\title{
The role of nutrition in the treatment of sarcopenia in Parkinson's disease: a scoping review
}

\author{
D. Brazier ${ }^{1}$, M. Ó. Breasail ${ }^{1}$, F.E. Lithander ${ }^{1,2}$ and E.J. Henderson ${ }^{1,2}$ \\ ${ }^{1}$ Population Health Sciences, Bristol Medical School, University of Bristol, Bristol, UK and \\ ${ }^{2}$ Older People's Unit, Royal United Hospitals Bath NHS Foundation Trust, Bath, UK
}

Sarcopenia is defined as an excessive loss of muscle mass and strength and associated with adverse outcomes including loss of physical function and frailty ${ }^{(1)}$. Parkinson's disease (PD) is a progressive neurodegenerative disease with motor and non-motor symptoms and people with PD (PwP) are particularly susceptible to gastro-intestinal non-motor symptoms and disease complications, which can lead to difficulty maintaining adequate nutrition. A combination of these symptoms and their underlying processes could lead to a higher prevalence of sarcopenia in $\mathrm{PwP}^{(2)}$. Despite the importance of nutrition in the management of sarcopenia, few studies have explored dietary interventions for the treatment of sarcopenia in PwP.

The aim of this scoping review was to summarise the available literature examining dietary intervention as a treatment for sarcopenia in PwP. Methods: A search of PubMed, CINAHL, MEDLINE, PsycINFO, AMED, EMBASE and Web of Science databases was performed up to 11/03/2021 to identify English language papers relating to sarcopenia and nutrition in PD using keywords "sarcopenia" and "Parkinson's disease". Results were screened by title and abstract in the first instance, then full text if required to ascertain eligibility.

233 articles were identified: three described nutrition as a treatment for sarcopenia in PwP. In a randomised controlled trial $(\mathrm{RCT})^{(3)}$, participants consumed a protein, leucine and vitamin D supplement alongside a four-week physical activity intervention, or a placebo with no exercise $(\mathrm{n}=150 \mathrm{PwP})$. At 30-day follow-up, the intervention group showed statistically significant improvements in strength and calf circumference. muscle mass was preserved. Energy and protein intake in the intervention group were higher, representing a limitation which could explain the beneficial results. A second $\mathrm{RCT}^{(4)}$, in inpatient older adults $(\mathrm{n}=127 ; \mathrm{n}=8$ PwP $[6.3 \%])$, investigated twice-daily whey protein supplementation $(40 \mathrm{~g} / \mathrm{d})$ in addition to physical rehabilitation programme lasting between four and eight weeks, and reported significant improvements in gait speed on discharge (mean difference, $+0.062 \mathrm{~m} / \mathrm{s} / \mathrm{month}[95 \% \mathrm{CI}, 0.043$ to 0.082$])^{(4)}$. The third article was a case report ${ }^{(5)}$ which described the "reversal of sarcopenia" in a patient with sarcopenic dysphagia following dietary and physical rehabilitation. There was heterogeneity in the definition of sarcopenia between the three studies. Additionally, as each combined both dietary and physical activity interventions the effect of diet per se is not known.

Evidence examining the role of nutrition in treatment of sarcopenia in $\mathrm{PwP}$ is limited. In older adults, there is an abundance of evidence positively supporting nutritional strategies which, alone or in combination with physical activity interventions, can slow progression of sarcopenia. The studies included in this review examined the combination of exercise and nutrition. For PwP, knowledge of how best to treat sarcopenia nutritionally, with consideration for levodopa absorption and meal timings would be beneficial as a focus for future research.

\section{References}

1. Cruz-Jentoft AJ, Bahat G, Bauer J, et al. (2019) Age Ageing 48(1), 16-31

2. Lima DP, de Almeida SB, de Carvalho Bonfadini J, et al. (2020) PLoS One 15(3), 1-12

3. Barichella M, Cereda E, Pinelli G, et al. (2019) Neurology 93(5), 485-496

4. Rondanelli M, Cereda E, Klersy C, et al. (2020) J Cachexia Sarcopenia Muscle 11(6), 1535-1547

5. Yamada Y, Shamoto H, Maeda K'\& Wakabayashi H (2018) Prog Rehabil Med 3 